\title{
Quantum order by disorder and accidental soft mode in $\operatorname{Er}_{2} \mathrm{Ti}_{2} \mathrm{O}_{7}$
}

\author{
M. E. Zhitomirsky, ${ }^{1}$ M. V. Gvozdikova, ${ }^{1}$ P. C. W. Holdsworth, ${ }^{2}$ and R. Moessner ${ }^{3}$ \\ ${ }^{1}$ Service de Physique Statistique, Magnétisme et Supraconductivité, \\ UMR-E9001 CEA-INAC/UJF, 17 rue des Martyrs, 38054 Grenoble Cedex 9, France \\ ${ }^{2}$ Laboratoire de Physique, École Normale Supérieure de Lyon, CNRS 69364 Lyon Cedex 07, France \\ ${ }^{3}$ Max-Planck-Institut für Physik komplexer Systeme, 01187 Dresden, Germany
}

(Dated: May 24, 2022)

\begin{abstract}
Motivated by recent neutron scattering experiments, we derive and study an effective "pseudodipolar" spin-1/2 model for the $X Y$ pyrochlore antiferromagnet $\operatorname{Er}_{2} \mathrm{Ti}_{2} \mathrm{O}_{7}$. While a bond-dependent in-plane exchange anisotropy removes any continuous symmetry, it does lead to a one-parameter 'accidental' classical degeneracy. This degeneracy is lifted by quantum fluctuations in favor of the noncoplanar spin structure observed experimentally - a rare experimental instance of quantum order by disorder. A non-Goldstone low-energy mode is present in the excitation spectrum in accordance with inelastic neutron scattering data. Our theory also resolves the puzzle of the experimentally observed continuous ordering transition, absent from previous models.
\end{abstract}

PACS numbers: 75.10.Jm, 75.30.Ds, 75.50.Ee,

Introduction.-Exotic low-temperature properties of magnetic pyrochlore oxides have their origin in the underlying frustrated lattice of corner-sharing tetrahedra, which is combined with diverse crystal-field effects for rare-earth ions 11. Initially, the attention was focused on to spin-ice materials $\mathrm{Ho}_{2} \mathrm{Ti}_{2} \mathrm{O}_{7}$ and $\mathrm{Dy}_{2} \mathrm{Ti}_{2} \mathrm{O}_{7}$ with Isinglike moments [2, 3] and on Heisenberg antiferromagnets $\mathrm{Gd}_{2} \mathrm{Ti}_{2} \mathrm{O}_{7}$ and $\mathrm{Gd}_{2} \mathrm{Sn}_{2} \mathrm{O}_{7}$ with large $S=7 / 2$ spins [4, 5]. The above pyrochlore compounds exhibit various kinds of ordered and unusual disordered classical magnetic phases naturally expected in view of large rare-earth moments. However, it was gradually recognized that collective behavior of low-energy magnetic doublets selected by the local crystalline electric field (CEF) for other rare-earth pyrochlores should be generally described by effective nonIsing pseudo-spin-1/2 Hamiltonians implying significant quantum effects [6 9].

$\mathrm{Er}_{2} \mathrm{Ti}_{2} \mathrm{O}_{7}$ has a special place in the family of magnetic pyrochlores. It is the only pyrochlore antiferromagnet found so far that exhibits strong easy-plane anisotropy with local moments confined to planes orthogonal to the local $\langle 111\rangle$-axes. Erbium Titanate has been extensively studied in the past and is well characterized experimentally [10 17. It orders below $T_{c} \simeq 1.2 \mathrm{~K}$, into an unusual $k=0$ non-coplanar antiferromagnetic structure shown in Fig. 1(a), the so called $\psi_{2}$ state [12, 13. This is rather surprising given that the geometry of the four local easy planes also allows coplanar spin arrangements such as the the $\psi_{3}$ state shown in Fig. 11(b), with the same energy as the noncoplanar state. Moreover, states obtained by reversing a pair of antiparallel spins in Fig. 1(b) minimize the dipolar energy [18].

Quantum order by disorder effect [19] was suggested as selection mechanism for the noncoplanar spin structure [12, because similar thermal order by disorder selection [20] indeed occurs in a model of classical $X Y$ moments [12, 21 23]. However, the concomitant tran-

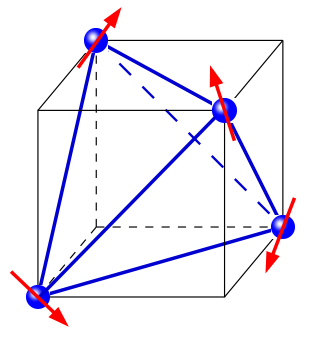

(a)

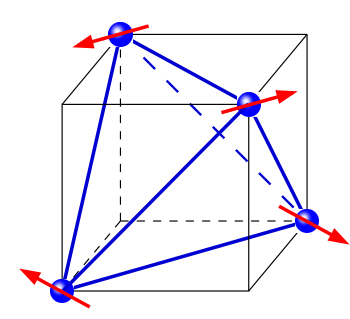

(b)
FIG. 1: (Color online) Possible $q=0$ spin structures of the $X Y$ pyrochlore antiferromagnet in zero applied field: $\psi_{2}$ state (a) and $\psi_{3}$ state (b).

sition turns out to be first order, in clear contradiction with the continuous second order transition observed in $\mathrm{Er}_{2} \mathrm{Ti}_{2} \mathrm{O}_{7}$ [10, 12]. Another recent experimental result, which has so far remained unexplained, is the presence of a quasi-acoustic mode in this highly anisotropic antiferromagnet found by inelastic neutron scattering (INS) measurements [14.

In this paper we formulate a minimal spin model for $\mathrm{Er}_{2} \mathrm{Ti}_{2} \mathrm{O}_{7}$ which accounts for this entire body of experimental results: quantum order by disorder stabilizes the observed ground state, with the low-energy mode in INS a remnant of the lifted degeneracy; while classical Monte Carlo simulations reproduce the continuous transition. Using this model, we analyze theoretically various physical effects such as the energetic selection due to quantum fluctuations, spin-wave results for the excitation spectrum and the nature of the finite-temperature phase transition studied by classical Monte Carlo simulations. We present various estimates of parameters in the model Hamiltonian, which has analogous form-but opposite sign! - to a dipolar interaction.

Model.-Free $\mathrm{Er}^{3+}$ ions $\left(J=15 / 2, g_{J}=6 / 5\right)$ have 
large magnetic moments $\mu=g_{J} \mu_{B} \sqrt{J(J+1)}=9.58 \mu_{B}$. A somewhat reduced value $\mu=8.9 \mu_{B}$ found in $\mathrm{Er}_{2} \mathrm{Ti}_{2} \mathrm{O}_{7}$ from high-temperature susceptibility measurements [11. reflects a partial quenching of the angular momentum by CEF. According to crystal-field analysis [12, 24, the ground-state Kramer's doublet $| \pm\rangle$ of $\mathrm{Er}^{3+}$ ions is strongly anisotropic:

$$
\left\langle \pm\left|J^{z}\right| \pm\right\rangle= \pm \frac{1}{2} \lambda_{z}, \quad\left\langle-\left|J^{-}\right|+\right\rangle=\left\langle+\left|J^{+}\right|-\right\rangle=\lambda_{\perp} .
$$

with $\lambda_{\perp}=6.35$ and $\lambda_{z}=0.202$. Neglecting higher CEF levels, one can use the operator equivalence

$$
J^{z}=\lambda_{z} S^{z}, \quad J^{x, y}=\lambda_{\perp} S^{x, y} .
$$

where $S^{\alpha}$ are components of an $S=1 / 2$ pseudo-spin defined in the subspace of the lowest doublet. Note that the operator relations $(2)$ are satisfied in the local coordinate frame for each magnetic ion.

In order to discuss possible forms of the effective Hamiltonian, it is instructive first to assume isotropic exchange between full magnetic moments, $\mathbf{J}_{i} \cdot \mathbf{J}_{j}$, and to project the nearest-neighbor (n.n.) exchange Hamiltonian onto the subspace of lowest CEF-doublets with the help of Eq. (2) 25]. Terms with $S_{i(j)}^{z}$ are further suppressed by a small factor $\lambda_{z} / \lambda_{\perp} \sim 10^{-2}$ and are neglected below. The remaining $X Y$ interactions are generally characterized by two parameters [26]

$$
\hat{\mathcal{H}}=\sum_{\langle i j\rangle}\left[\mathcal{J} \mathbf{S}_{i}^{\perp} \cdot \mathbf{S}_{j}^{\perp}+\mathcal{J}_{a}\left(\mathbf{S}_{i}^{\perp} \cdot \hat{\mathbf{r}}_{i j}\right)\left(\mathbf{S}_{j}^{\perp} \cdot \hat{\mathbf{r}}_{i j}\right)\right],
$$

where $\hat{\mathbf{r}}_{i j}=\left(\mathbf{r}_{i}-\mathbf{r}_{j}\right) /\left|\mathbf{r}_{i}-\mathbf{r}_{j}\right|$. The first term is isotropic exchange between transverse components of n.n. spins confined to the local $\{111\}$ planes. The second term represents bond-dependent exchange anisotropy. It has a pseudo-dipolar form but both signs of $\mathcal{J}_{a}$ are allowed by symmetry. Although the above projection procedure yields vanishing $\mathcal{J}_{a}$, the in-plane exchange anisotropy may be generated by virtual excitations into higher CEF levels [25] or into states with different occupancy on rareearth ions [7. As we shall see below, it is essential to include $\mathcal{J}_{a}$ in the effective Hamiltonian (3), since quantum fluctuations are singular in the $\mathcal{J}_{a} \rightarrow 0$ limit.

Ground-state selection.-The classical ground states of the Hamiltonian (3) with $\mathcal{J}_{a}=0$ satisfying the constraint $\sum_{\text {tet }} \mathbf{S}_{n}^{\perp}=0$ form an infinite but nonextensive set of states, producing planes of zero-energy modes in the excitation spectrum [22]. Finite anisotropy $\mathcal{J}_{a} \neq 0$ almost eliminates this degeneracy. For $\mathcal{J}_{a}>0$, we find that the classical energy (3) is minimized for a subset of $k=0$ states. Two of them, so called $\psi_{2}$ and $\psi_{3}$ states [13, are shown in Fig. 1, while other spin configurations are constructed as their linear combination:

$$
\mathbf{S}_{n}(\varphi)=S\left(\hat{\mathbf{x}}_{n} \cos \varphi+\hat{\mathbf{y}}_{n} \sin \varphi\right), \quad n=1 \ldots 4
$$

such that $\varphi=0(\pi / 2)$ corresponds to $\psi_{2}\left(\psi_{3}\right)$, respectively. Thus, instead of an infinite number of variables, the classical ground-states of Eq. 3 for $\mathcal{J}_{a}>0$ are parameterized by one global continuous angle, $\varphi$.

Spin states $\psi_{2}$ and $\psi_{3}$ form a basis of the twodimensional irreducible representation $E$ of the tetrahedral point group. They transform as $\psi_{2} \sim 3 z^{2}-r^{2}$ and $\psi_{3} \sim x^{2}-y^{2}$ under cubic rotations. Consequently, the two spin configurations have the same mean-field energies for any extension of the Hamiltonian (3), involving only bilinear spin-spin interactions (e.g., further-neighbor exchange or long-range dipolar interactions). The meanfield degeneracy may be lifted only by interactions of sixth order in spin components [27, though these are not explicitly present in the effective Hamiltonian since $S=1 / 2$. Nevertheless, such interactions may be generated dynamically by thermal or quantum fluctuations.

To study the effect of quantum fluctuations on the remaining continuous degeneracy we use harmonic spinwave theory. For this, spin operators in the effective Hamiltonian (3) are assumed to have an arbitrary spin $S$ and the usual $1 / S$ expansion is applied, substituting $S=1 / 2$ in the final expressions. This standard approach yields the following results.

Magnon excitation energies for an arbitrary classical ground-state (4) can be expressed as

$$
\varepsilon_{\mathbf{k}}^{(n)}=\mathcal{J} S \sqrt{\left(2+j_{a}\right)^{2}+2\left(2+j_{a}\right) \lambda_{\mathbf{k}}^{(n)}},
$$

where, $j_{a}=\mathcal{J}_{a} / \mathcal{J}$ and $\lambda_{\mathbf{k}}^{(n)}$ are four eigenvalues of the matrix $C_{\mathbf{k}}$,

$$
C_{\mathbf{k}}=\left(\begin{array}{cccc}
0 & c_{x y}^{+} & c_{y z}^{+} & c_{x z}^{+} \\
c_{x y}^{+} & 0 & c_{x z}^{-} & c_{y z}^{-} \\
c_{y z}^{+} & c_{x z}^{-} & 0 & c_{x y}^{-} \\
c_{x z}^{+} & c_{y z}^{-} & c_{x y}^{-} & 0
\end{array}\right)
$$

with $c_{x y}^{ \pm}=t_{x y} \cos \left(k_{x} \pm k_{y}\right) / 4, \ldots$, and

$$
\begin{aligned}
t_{x y} & =\frac{4}{3} \sin (\varphi-\pi / 3) \sin (\varphi+\pi / 3)-\frac{j_{a}}{3} \sin ^{2} \varphi, \\
t_{y z} & =-\frac{4}{3} \sin \varphi \sin (\varphi-\pi / 3)-\frac{j_{a}}{3} \sin ^{2}(\varphi+\pi / 3), \\
t_{x z} & =-\frac{4}{3} \sin \varphi \sin (\varphi+\pi / 3)-\frac{j_{a}}{3} \sin ^{2}(\varphi-\pi / 3) .
\end{aligned}
$$

The quantum correction to the ground-state energy normalized per spin is

$$
\Delta E_{\text {g.s. }}=\frac{1}{8} \sum_{n=1}^{4} \int \frac{\mathrm{d}^{3} k}{V_{\mathrm{BZ}}} \varepsilon_{\mathbf{k}}^{(n)}-\frac{1}{2} \mathcal{J} S\left(2+j_{a}\right),
$$

where $V_{\mathrm{BZ}}$ is the Brillouin zone volume. $\Delta E_{\text {g.s. }}(\varphi)$ is shown in the inset of Fig. 2 for $S=1 / 2$, exhibiting a clear minimum for the noncoplanar $\psi_{2}$ state $(\varphi=0, \pi / 3, \ldots)$ for any value of $j_{a}$. Hence quantum fluctuations, like thermal fluctuations, select the $\psi_{2}$ state from the ensemble of classical ground states giving an example of quantum order by disorder. A similar calculation for isotropic 


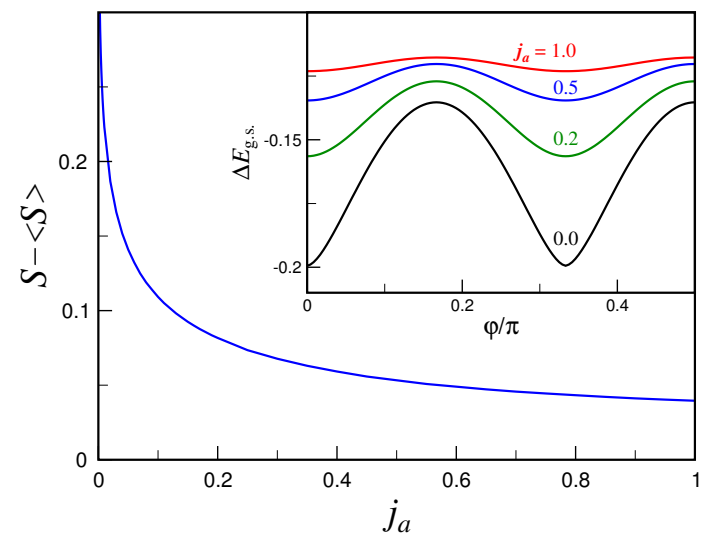

FIG. 2: (Color online) Inset: the ground-state energy in units of $\mathcal{J}$ for degenerate classical configurations parameterized by angle $\varphi$, see Eq. (4). Main panel: the quantum spin reduction in the noncoplanar state $\psi_{2}$ versus the bond-dependent exchange anisotropy parameter $j_{a}=\mathcal{J}_{a} / \mathcal{J}$.

exchange $j_{a}=0$, was recently presented in [23]. However, as shown below, quantum fluctuations become singular and essentially nonperturbative in the isotropic case and may completely destroy the long-range magnetic order.

Harmonic spin-wave theory also yields the leading quantum correction to the value of the ordered moment $\langle S\rangle$. Most conveniently this can be done with the help of a fictitious staggered field $h_{s} \rightarrow 0$. The corresponding expression for $\delta S=S-\langle S\rangle$ is

$$
\delta S=\frac{1}{8} \sum_{n=1}^{4} \int \frac{\mathrm{d}^{3} k}{V_{\mathrm{BZ}}} \frac{2+j_{a}+\lambda_{\mathbf{k}}^{(n)}}{\sqrt{\left(2+j_{a}\right)^{2}+2\left(2+j_{a}\right) \lambda_{\mathbf{k}}^{(n)}}}-\frac{1}{2} .
$$

The spin reduction $\delta S$ in the ordered $\psi_{2}$ state (Fig. 2) diverges logarithmically as $j_{a} \rightarrow 0$. This is a result of the effective dimensional reduction to one dimensional behavior, due to the planes of zero-energy modes in the magnon dispersion $\varepsilon_{\mathbf{k}}$ in the isotropic case 22]. However, a small anisotropy of $j_{a} \simeq 0.05$ already cuts off the singularity and restores the perturbative nature of the quantum correction. The measured ordered moment of $\mu=3.2 \mu_{B}$ in $\mathrm{Er}_{2} \mathrm{Ti}_{2} \mathrm{O}_{7}$ 13 indicates a $16 \%$ renormalization from the nominal value $\mu_{0} \approx 3.8 \mu_{B}$ for the $\mathrm{Er}^{3+}$ ground-state doublet [12. This gives an estimate of $j_{a} \sim 0.3-0.5$. The large uncertainty is related to the unknown precision for $\mu_{0}$, which cannot be measured directly, but our analysis is consistent with a perturbation of considerable size.

Excitation spectrum.-With both Hamiltonian and its ground state in hand, we compute the spin-wave spectrum in $\mathrm{Er}_{2} \mathrm{Ti}_{2} \mathrm{O}_{7}$. The $\psi_{2}$ magnetic structure allows six different domains with $\varphi=\pi n / 3$. We choose $\varphi=0$ (pictured in Fig. 1(a)) with spins pointing along $[ \pm 1, \pm 1, \pm 2]$ cubic directions. Analytical expressions for magnon energies can be derived from Eq. (5) for a few symmetry directions in the Brillouin zone. For $\mathbf{k}=(q, 0,0)$, the four branches are

$$
\left(\frac{\varepsilon_{\mathbf{k}}}{\mathcal{J} S}\right)^{2}=\left(2+j_{a}\right)\left[2+j_{a} \mp 2 \cos \frac{q}{4} \pm \frac{1}{2} j_{a}\left(1+\cos \frac{q}{4}\right)\right] \text {. }
$$

Along the $\mathbf{k}=(0,0, q)$ line, the two dispersive branches are expressed as

$$
\varepsilon_{\mathbf{k}}=\mathcal{J} S \sqrt{\left(2+j_{a}\right) j_{a}[1 \pm \cos (q / 4)]}
$$

while the two other modes remain dispersionless with $\varepsilon_{\mathbf{k}}=\mathcal{J} S \sqrt{\left(2+j_{a}\right)\left(4+j_{a}\right)}$.

The characteristic low-energy feature of the calculated spectra is a gapless mode with acoustic dispersion $\varepsilon_{\mathbf{k}} \approx c k$ for $k \rightarrow 0$. This remarkably reproduces a variety of experimental results for $\mathrm{Er}_{2} \mathrm{Ti}_{2} \mathrm{O}_{7}$ including the $T^{3}$-dependence of the heat capacity [10], the inelastic neutron data 14, and the electron spin resonance measurements [15. The presence of the gapless branch in the harmonic approximation is directly linked to the classical degeneracy of the model (3) and is, therefore, accidental in the sense that is not related to any symmetry of the magnetic system. Hence, a finite gap will generically be produced by quantum fluctuations in higher orders of the spin-wave theory. Corresponding calculations are rather cumbersome and are not attempted here. We only remark that the generated quantum gap should depend on the curvature of $\Delta E_{\text {g.s. }}(\varphi)$ in the vicinity of its minimum. Since the minimum becomes more shallow with increasing anisotropy, see the inset of Fig. 2, we expect that for $j_{a} \geq 0.5$ the actual gap may be rather small.

Figure 3 shows the dispersion of four magnon branches in the [100] direction in comparison with the inelastic neutron scattering data of Ruff et al. 14] scanned from their Fig. 4. We find particularly good agreement between theory and experiment for the following values of the microscopic parameters: $\mathcal{J}=0.28 \mathrm{meV}$ and $j_{a}=1$, corresponding curves are plotted by solid lines. Magnon

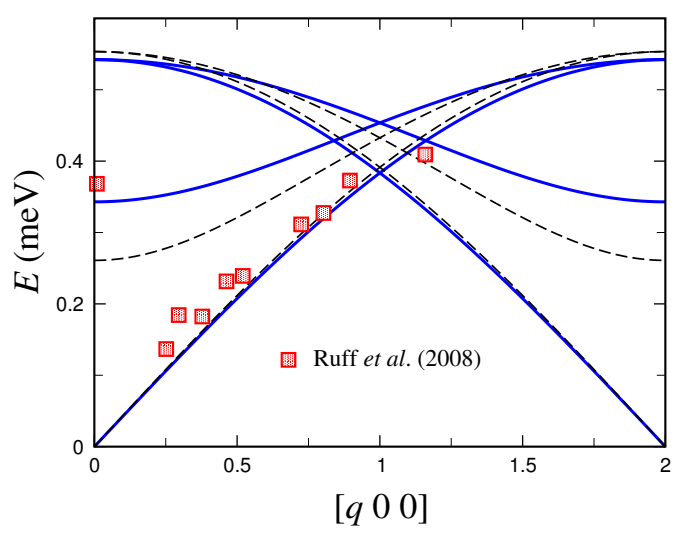

FIG. 3: (Color online) Zero-field dispersion of magnons in $\mathrm{Er}_{2} \mathrm{Ti}_{2} \mathrm{O}_{7}$ along the [100] direction. Wave-vectors are measured in units of $2 \pi$. Solid lines are calculated for $\mathcal{J}=$ $0.28 \mathrm{meV}$ and $j_{a}=1$, while dashed lines are for $\mathcal{J}=0.33 \mathrm{meV}$ and $j_{a}=0.5$. Open squares are experimental data points 14 . 


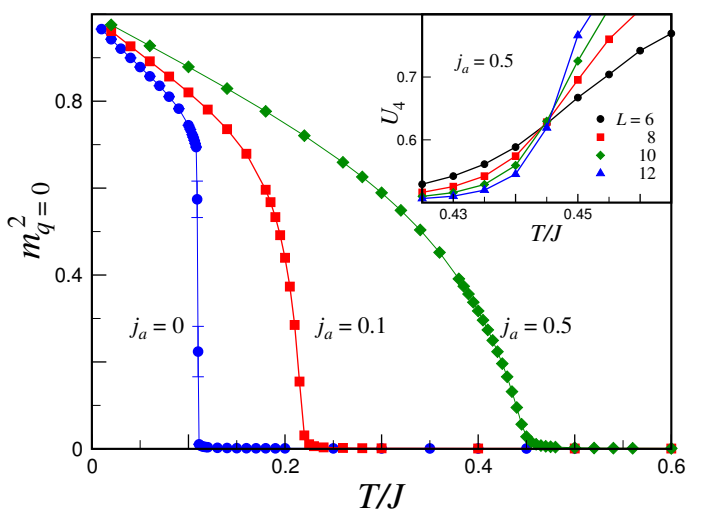

FIG. 4: (Color online) Main panel: temperature dependence of the sublattice magnetization for the classical $X Y$ pyrochlore antiferromagnet with in-plane anisotropic exchange on periodic cluster with $L=12$. Inset: the fourth-order Binder cumulant for several lattice sizes at fixed $j_{a}=0.5$.

energies for $\mathcal{J}=0.33 \mathrm{meV}$ and $j_{a}=0.5$ a value extracted from the reduced moment (see above) are shown by dashed lines. While the low-energy branch is fitted equally well, a larger $j_{a}$ is favored by the position of the lower gap $\Delta \approx 0.38 \mathrm{meV}$.

We can extract further information about the the microscopic parameters from values of the critical field between the antiferromagnetic phase and the polarized paramagnetic state [14-17. The high-field polarized states are particularly simple for field orientations along [100] and [110] axes. Repeating the above spin-wave calculations for $\mathbf{H} \|$ [110] we obtain the critical field from the vanishing gap in the magnon spectrum

$$
g_{\perp} \mu_{B} H_{c}^{[110]}=\mathcal{J} S\left[4+\frac{4}{\sqrt{3}}+j_{a}\left(\frac{2}{\sqrt{3}}-1\right)\right],
$$

where $g_{\perp}=g_{J} \lambda_{\perp} \approx 7.6$. The dependence of 12 on $j_{a}$ is very weak and from the extrapolated $T=0$ value $H_{c}^{[110]} \simeq 1.8 \mathrm{~T}$ [14 16], we derive $\mathcal{J} \simeq 0.25 \mathrm{meV}$, which is consistent with the estimate based on neutron data. Additional measurements of the excitation spectrum and/or the angular dependence of the critical field $H_{c}$ at low temperatures should allow a more precise identification of the microscopic parameters of the model (3) in the case of $\mathrm{Er}_{2} \mathrm{Ti}_{2} \mathrm{O}_{7}$.

Finite-temperature transition.-We finally consider the nature of ordering transition in the $X Y$ pyrochlore antiferromagnet with the bond-dependent exchange anisotropy. Since the original quantum model (3) remains intractable for numerical investigation, we study instead a simplified version of (3) with spins represented by classical unit vectors. The classical Monte Carlo simulations were performed on periodic lattices of $N=4 L^{3}$ spins with linear sizes $L=6-12$ using the Metropolis algorithm with $\sim 10^{7}$ Monte Carlo steps. Temperature dependence of the sublattice magnetization $m_{q=0}$ for antiferromagnetic ordering with zero wave-vector is shown in Fig. 4 for three values of $j_{a}$. For the model with isotropic exchange, $j_{a}=0$, a clear jump appears at $T_{c} / J=0.110(1)$. However, already a small exchange anisotropy $j_{a}=0.1$ changes the behavior $m_{q=0}(T)$ to continuous.

This is confirmed by the temperature dependence of the fourth-order Binder cumulant

$$
U_{4}=\frac{\left\langle m_{q=0}^{4}\right\rangle}{\left\langle m_{q=0}^{2}\right\rangle^{2}} .
$$

According to finite-size scaling theory, Binder cumulants for clusters with different linear sizes $L$ cross at the critical point where spin-spin correlations exhibit a power-law decay. The crossing point $T_{c} / J=0.445(2)$ for $j_{a}=0.5$ (Fig. (4) gives an accurate estimate for the transition point in this case. In our Monte Carlo simulations we also investigated various Potts-type order parameters, which distinguish between the noncoplanar $\psi_{2}$ and the planar $\psi_{3}$ spin structure. As $T \rightarrow 0$ there is a clear selection of the $\psi_{2}$ configuration. However, at higher temperatures spins strongly fluctuate restoring at $T \rightarrow T_{c}$ the continuous $X Y$ degeneracy parameterized by angle $\varphi$ in (4).

In conclusion, we have proposed a simple quantum model for the $X Y$ pyrochlore antiferromagnet $\mathrm{Er}_{2} \mathrm{Ti}_{2} \mathrm{O}_{7}$, which includes bond-dependent in-plane exchange anisotropy of strength $j_{a}$. We show that the order by disorder selection occurring for $j_{a}=0$ is maintained at both quantum and classical level for perturbations up to at least the strength of the isotropic exchange. The exchange anisotropy also reproduces the observed secondorder transition found in $\mathrm{Er}_{2} \mathrm{Ti}_{2} \mathrm{O}_{7}$ in thermodynamic measurements. Further work is planned to investigate field evolution of the antiferromagnetic state and role of the long-range part of the dipolar interactions, but it is worth noting here that the perturbation is of the same form, but of opposite sign to the dipolar interaction for n.n. pairs of spins. As noted by Stasiak et al. 23], an exchange anisotropy of this kind can therefore overcome the n.n. part of the dipolar interaction. In addition, the longrange part of the dipolar interaction loses importance as the tetrahedral units have no net magnetic moment in the ground state. These observations provide a possible explanation as to why the dipolar effects appear to be absent in $\mathrm{Er}_{2} \mathrm{Ti}_{2} \mathrm{O}_{7}$.

The fact that our theoretical analysis with single parameter $j_{a}$ is in agreement with many experimental features of $\mathrm{Er}_{2} \mathrm{Ti}_{2} \mathrm{O}_{7}$ strongly supports the above picture and in the process provides strong evidence that the ordering transition and the stability of the experimentally observed noncoplanar magnetic structure is indeed a remarkable experimental realization of the order by disorder mechanism [12. A consequence of this is the unique appearance of a non-Goldstone acoustic magnon branch with a very small gap. 
We are grateful to Z. Hao and S. Sosin for their help at the initial stage of this project. We thank S. Bramwell, P. Dalmas de Réotier, B. Gaulin, M. Gingras, and S. Sosin for many fruitful discussions. MEZ and PCWH acknowledge hospitality of the Max Planck Institute for the Physics of Complex Systems, where part of this work has been done.

Note added in proof.-After submission of this work we became aware of a related preprint by Savary et al. $28]$.

[1] J. S. Gardner, M. J. P. Gingras, and J. E. Greedan, Rev. Mod. Phys. 82, 53 (2010).

[2] M. J. Harris, S. T. Bramwell, D. F. McMorrow, T. Zeiske, and K. W. Godfrey, Phys. Rev. Lett. 79, 2554 (1997).

[3] R. Moessner, Phys. Rev. B 57, 5587(R) (1998).

[4] N. P. Raju, M. Dion, M. J. P. Gingras, T. E. Mason, J. E. Greedan, Phys. Rev. B 59, 14489 (1999).

[5] A. S. Wills, M. E. Zhitomirsky, B. Canals, J.-P. Sanchez, P. Bonville, P. Dalmas de Réotier, and A. Yaouanc, J. Phys.: Condens. Matter 18, L37 (2006).

[6] H. R. Molavian, M. J. P. Gingras, and B. Canals, Phys. Rev. Lett. 98, 157204 (2007).

[7] S. Onoda and Y. Tanaka, Phys. Rev. Lett. 105, 047201 (2010).

[8] K. A. Ross, L. Savary, B. D. Gaulin, and L. Balents, Phys. Rev. X 1, 021002 (2011).

[9] L. Savary and L. Balents, Phys. Rev. Lett. 108, 037202 (2012)

[10] H. W. Blöte, R. F. Wielinga, W. J. Huiskamp, Physica 43, 549 (1969).

[11] S. T. Bramwell, M. N. Field, M. J. Harris, and I. P. Parkin, J. Phys.: Condens. Matter 12, 483 (2000).

[12] J. D. M. Champion, M. J. Harris, P. C. W. Holdsworth, A. S. Wills, G. Balakrishnan, S. T. Bramwell, E. Cizmar, T. Fennell, J. S. Gardner, J. Lago, D. F. McMorrow, M.
Orendac, A. Orendacova, D. McK. Paul, R. I. Smith, M. T. F. Telling, and A. Wildes, Phys. Rev. B 68, 020401(R) (2003).

[13] A. Poole, A. S. Wills, and E. Lelièvre-Berna, J. Phys.: Condens. Matter 19, 452201 (2007).

[14] J. P. C. Ruff, J. P. Clancy, A. Bourque, M. A. White, M. Ramazanoglu, J. S. Gardner, Y. Qiu, J. R. D. Copley, M. B. Johnson, H. A. Dabkowska, and B. D. Gaulin, Phys. Rev. Lett. 101, 147205 (2008).

[15] S. S. Sosin, L. A. Prozorova, M. R. Lees, G. Balakrishnan, and O. A. Petrenko, Phys. Rev. B 82, 094428 (2010).

[16] H. B. Cao, I. Mirebeau, A. Gukasov, P. Bonville, and C. Decorse, Phys. Rev. B 82, 104431 (2010).

[17] O. A. Petrenko, M. R. Lees, and G. Balakrishnan, J. Phys.: Condens. Matter 23, 164218 (2011).

[18] S. E. Palmer and J. T. Chalker, Phys. Rev. B 62, 488 (2000).

[19] E. F. Shender, Zh. Eksp. Teor. Fiz. 83, 326 (1982) [Sov. Phys. JETP 56, 178 (1982)].

[20] For a discussions of different types of order by disorder, see J. Villain, R. Bidaux, J. P. Carton and R. Conte, J. Physique 41, 1263 (1980); C. L. Henley, Phys. Rev. Lett. 62, 2056 (1989); R. Moessner, Can. J. Phys. 79, 1283, (2001).

[21] S. T. Bramwell, M. J. P. Gingras, and J. N. Reimers, J. Appl. Phys. 75, 5523 (1994).

[22] J. D. M. Champion and P. C. W. Holdsworth, J. Phys.: Condens. Matter 16, S665 (2004).

[23] P. Stasiak, P. A. McClarty, and M. J. P. Gingras, arXiv:1108.6053

[24] P. Dasgupta, Y. Jana, and D. Ghosh, Solid State Commun. 139, 424 (2006).

[25] H. Shiba, Y. Ueda, K. Okunishi, S. Kimura, and K. Kindo, J. Phys. Soc. Jpn. 72, 2326 (2003).

[26] S. H. Curnoe, Phys. Rev. B 78, 094418 (2008).

[27] P. A. McClarty, S. H. Curnoe, and M. J. P. Gingras, J. Phys.: Conf. Ser. 145, 012032 (2009).

[28] L. Savary, K. A. Ross, B. D. Gaulin, J. P. C. Ruff, and L. Balents, arXiv:1204.1320. 